\title{
Research on the New Power Quality Online Monitoring and Analysis System Design Based on the LABview
}

\author{
Mengmei Ren ${ }^{1, a}$ \\ ${ }^{1}$ Anyang Vocational and Technical College, Anyang, Henan, China, 455000
}

\begin{abstract}
Keywords: Real-Time Monitoring, Transient Power Quality, Virtual Instrument, Generalized S Transform, Data Compression
\end{abstract}

\begin{abstract}
Power Quality Improvement premise is to obtain accurate power quality real-time information. In order to achieve the targets more accurate real-time monitoring of power quality, paper LabVIEW graphical software platform for the development, design real-time power quality monitoring and analysis system, using generalized S transform and combining tree classification rules implemented method of transient power quality disturbance signal time targeting and automatic identification. The system includes a parameter setting, data acquisition and analysis, recorder storage and data transfer 4 function modules with real-time monitoring and recording various indicators steady power quality disturbances transient analysis time of occurrence and recognition. Programmable three-phase AC power by the power quality analog signal, the system measurement function experiments and the results showed that: RMS voltage error is less than $05 \%$ and a frequency error is less than $001 \mathrm{~Hz}$, each harmonic voltage error. There are less than 5\%, and transient voltage oscillation disturbance detection interrupt signal value exist errors, but errors are within $1 \mathrm{~ms}$ and the validity and effectiveness of the system. The system can function fully meet the application requirements of power quality monitoring and analysis, can provide the necessary data to support the development of improved power quality and power quality management related measures, with good practical significance.
\end{abstract}

\section{Introduction}

Electricity as a commodity, how to ensure and improve power quality, so as to ensure normal production and life, has become an important topic in the field of domestic and foreign electrician urgent need to address. To solve this problem, we must first power quality to make timely and accurate detection, and then take appropriate control methods and strategies, targeted to improve the quality of power to meet the electricity needs of users, it is possible to power quality timely and accurate detection is very important.

For power quality monitoring of problems, a lot of scholars to carry out research work, mainly focused on the design and implementation of theoretical research and monitoring devices in the detection algorithm. In recent years, transient power quality detection algorithms and data compression methods research has made great progress, many algorithms in other areas also widely used. Domestic late start, power quality monitoring devices, according to the detection method can be divided into the portable power quality analyzer and line power quality monitoring device, wherein the portable power quality analyzer is mainly applied to the field of special tests, cannot grasp the long-term operation of the grid situation. At present, power quality monitoring is aimed at a more comprehensive understanding of power quality level, providing reliable data basis for the development of specific measures to improve power quality and control pollution of the power grid, so online power quality measuring instrument rapidly in recent years is widely used in electricity quality monitoring. Looking at the current applied to the analysis of power quality monitoring equipment, mostly digital processor (DSP) technology, but with the power quality test equipment continued to digital, integrated, networked, intelligent direction, the traditional signal detection device insufficient analysis, data transmission, data storage and other aspects gradually reflected.

A new generation of software-centric technology --- instruments virtual instrument technology, completely changed the traditional hardware-centric measurement concept cannot be limited hardware resources, flexible expansion and upgrades to achieve measurement functions, can make 
full use of computer itself powerful hardware and software resources to achieve set of data processing, display, transmission, storage and other integrated intelligent measurement systems, has become a new generation of measurement, control the development direction of system development. According to the current problems of power quality monitoring, this paper based on virtual instrument technology, LabVIEW development environment Power Quality Monitoring and Analysis Department of the company's NI

EC aims to achieve steady-state real-time monitoring of power quality indicators and transient power quality disturbances, provide a theoretical basis and data support for the development of power quality control measures. This paper studies the design of real-time monitoring system of power quality has important practical significance.

\section{The Overall Structure of Monitoring System}

Based on the overall structure of power quality virtual instruments, real-time monitoring and analysis system, including hardware and software components, hardware includes signal conditioning circuitry, data acquisition card and equipped with virtual instrument software, computer; software part that LabVIEW implemented monitoring functions, including data processing, data storage, data transfer, and statistical analysis.

Hardware Configuration. Since monitoring of the original voltage and current signals for the secondary circuit of the three-phase voltage, current and neutral voltage signal relative to the rated voltage is generally $100 \mathrm{~V}$, rated phase current is generally $5 \mathrm{~A}$ or $1 \mathrm{~A}$, and therefore must pass in order to signal conditioning into the data acquisition card. Signal conditioning circuit design of the system AC voltage and current signals into $-5-5 \mathrm{~V}$ voltage signal can access the data acquisition card, for signal conditioning circuit includes a choice of sensors and anti-aliasing low-pass filter. The design uses the Swiss Lyme (LEM) company LV 25-P voltage sensor and current sensor LA 25-NP. In the primary and secondary side voltage and current sensor requires a precise sampling resistor in series, this design are used $100 \Omega$ precision sampling resistor. The sensor has high accuracy, good linearity, strong anti-interference ability, excellent common-mode rejection ratio and high insulation properties strong, suitable for signal conditioning power quality monitoring system.

According to the sampling theorem, the sampling frequency must be greater than the maximum frequency of the analog signal twice, but the actual broadband noise may be disturbed, therefore, to ensure that the sampling process does not produce aliasing, design an anti-aliasing low-pass filter. As the design for up to 50 harmonics (default is 19 harmonics) detection, detection and analysis of the need for the signal component $0 \sim 2.5 \mathrm{kHz}$, the sampling frequency of power quality monitoring system is set to $12.8 \mathrm{kHz}$, so that oversampling can the elimination of aliasing play a role, so the design uses a cutoff frequency of $5 \mathrm{kHz}$ second-order low-pass digital filter that uses LabVIEW Butterworth filter in the sub-VI. The card comes with a $250 \mathrm{kHz}$ sampling rates up to 16 -bit A / D converter provides 16 single-ended analog inputs or 8 differential analog input channels that supports PCI bus DMA function, multi-threaded, high-speed data transmission and no interval of analog inputs. The PCI-1716L is inserted into the computer's PCI worse, install hardware drivers and LabVIEW drivers and libraries, which can connect the data acquisition card and LabVIEW software. Computer selected rackmount industrial computer, with good stability, strong anti-interference characteristics, the standard formula for the width of the shelves are easy to place in the substation.

Software Composition. This design software configuration diagram Power Quality Monitoring and Analysis System. The main module consists of four parts. Among them, the parameter setting module is used to set permissions management and acquisition parameters, including the total number of points a sampling frequency, sampling period, PT ratio, CT ratio, the more the index of alarm limit parameters; data acquisition and analysis module completes the data arithmetic processing, storage module complete record wave based on the data written to the database and read, the data transmission module for communication function of the network, so that the upper-level monitoring computer or server to receive collected data transmission, and has a function of printing reports . 


\section{System Software Design}

Parameter Setting Module. Based on LabVIEW real-time power quality monitoring and analyzing system, the parameter settings including sample parameter settings and the alarm limit parameter setting its tab interface shown in Figure 3, wherein the sampling parameter settings including the sampling frequency, the sampling window points buffer area size, voltage transformer ratio, voltage and current transformer ratio, select the current of each channel signal, each channel has a Boolean control is enabled, Boolean true elected, said sampling function of the channel open. For more limited set of parameters, including setting limits steady power quality indicators, setting national standards in accordance with the predetermined value, when the need to monitor the current harmonics is exceeded, the need to set the actual short-circuit capacity, that is, the common connection point line monitoring minimum short-circuit capacity, then the software automatically based on the harmonic current allowable value correction formula to calculate the allowed values of harmonic current injection, the design of the system default calculation 2-19 times harmonic current allowable value.

Data Collection and Analysis Module. A selection of data acquisition card PCI-1716L has a 1 $\mathrm{K} / \mathrm{D}$ sampling FIFO buffer, and having the ability to direct memory access (Directmemory access, DMA), the DMA bus data transfer does not require CPU intervention, directly through the PCI bus transfer data to RAM in order to achieve high-speed data throughput.

Data acquisition card installation disc in the band have the LabVIEW DAQ (data acquisition) driver program, after installation to the next directory LabVIEW, open the VI block diagram interface, you can select the capture card device LabVIEWDAQ sub VI in the user library. 4, the high-speed data acquisition and the main advantage of the circular buffer queue (Queue) technology, in addition to setting the buffer size of the data buffer, there is a queue buffer is a FIFO queue structure the structure can ensure an orderly transfer of data. Data analysis includes the detection module, the steady-state and transient disturbance index detection Detect and transient power quality disturbances steady-state power quality indicators for different sub-VI, and the program is executed in parallel multi-threaded, additional sub-attributes of VI can be set to re-enter (reentrant) sub-VI, not only can guarantee parallelism call, you can also make calls everywhere have maintained their status. Transient disturbance trigger module rms calculation method, according to sampling frequencies, whichever half period moving average processing sampled data, the RMS value exceeds the threshold as the basis for judging whether a transient disturbance. Programming threshold value is 0.1 p. U., With reference to a valid value for RMS calculation results on a queue element. Suppose the queue element in the effective value VRMS, compute the rms value of the first slide +1 queue element, when there is more than one RMS value of $110 \%$ or less than $90 \%$ VRMS VRMS, the trigger disturbance detection module; when there is no rms value exceeds the threshold value, the effective value of the first element of the queue +1 written VRMS, as a reference value of the first element of the queue +2 .

Recorder Memory Module. Disturbance oscillography memory module includes storage compression waveforms reconstructed waveform query, historical records and other indicators, the module database to store, manage, and query data. Among them, the power quality recording data compression using the image smoothing algorithm to enhance the two-dimensional wavelet transform encoding algorithm and image SPITH format has better compression performance, which greatly reduces the storage space. LabVIEW and the database connection is most commonly used on the ADO (ActiveX Data Object) technology, due to the Windows operating system already contains ADO components and ADO with the ODBC (Open

Database Connectivity, open data interconnect) connection can access any supported ODBC database, such as SQL Server, Oracle, MySQL, FoxPro, Microsoft Access, etc. The design selected by

SQL Server 2000 as a real-time power quality monitoring and analyzing system of back-end database. LabVIEW provides access to ADO-based database technology package LabSQL asked, after installation can directly call LabSQL library sub-VI ADO perform basic operations, and execute SQL statements. Need to establish its own database in ODBC data source management tool 
under Windows before using the database, the database name is called named data source name DSN (Data Source Name), the database read and write operations in the future are to DSN of identity. To improve database storage, query efficiency of large amounts of data, database design using multiple tables to save different power quality parameters.

Data Transfer Module. Data transmission module to achieve functional data communication network, LabVIEW in network communication technology including shared variables, DataSocket, TCP / IP, UDP, remote panel recalls. This design uses LabVIEW Web server technology and you can display and control the local VI front panel on the remote computer. Firstly, it needs to connect remote server configuration and start the LabVIEW Web server, and then call the client computer to install the server version of the same engine LabVIEWRun-Time, so you can connect to a remote VI customer service side panel via the web, and set permissions through remote control operations.

\section{Experimental Verification}

In order to verify the effectiveness of the design of real-time power quality monitoring and analysis system, programmable through a three-phase AC power supply (The Chroma61703 Programmable AC Power Source) to simulate three phase power frequency voltage signal and the analog signal harmonics and transient disturbance signals related test, installed in a computer data acquisition card with the experimental test platform.

When testing the software parameter setting voltage transformer ratio is set to 1 , consistent with the value thus measured RMS value of the AC power output for easy comparison error; sampling frequency default $128 \mathrm{kHz}$.. On any one phase of the AC voltage harmonic signal is added, each harmonic voltage Results Monitoring System software testing.

According to the test results that (1) This design of real-time power quality monitoring and analyzing system can correct and effective measure amplitude and frequency of the signal power quality, meet the national standard GB / T 19862-2005 "General requirements for power quality monitoring equipment" in the class a instrument error is less than $0.5 \%$ rms voltage, frequency error is less than $0.01 \mathrm{~Hz}$, each harmonic voltage error is less than 5\%. (2) As the content of higher harmonics decreases, the resulting measurement error also increases, wherein the error value 11 times harmonic voltage reached $4.19 \%$, mainly by the hardware system signal conditioning and data acquisition interference circuit caused the error. For hardware anti-jamming technology, including shielding, filtering, grounding and power jamming four aspects, such as adding an active filter; signal acquisition using differential inputs wiring; avoid ground loops, parallel single-point ground to reject common mode interference. In List Mode Programmable AC power analog voltage swells, voltage sag, voltage interruption, a common transient power quality disturbance signal transient oscillation, generalized $\mathrm{S}$ transform and classification tree method implementation rules transient power quality disturbances signal time positioning and automatic identification of transient disturbance signal detection result shows: mutation detection signal disturbance has good accuracy, can effectively locate the start time and end time the disturbance occurred. Wherein the disturbance time voltage swells and sags detection signal values are identical with the set value, can accurately detect the beginning and ending time of the disturbance occurred, and the interruption and transient voltage oscillation signal detected value of disturbance there is an error, but the error in within $1 \mathrm{~ms}$, indicating that the system has good positioning accuracy.

\section{Conclusion}

Through LabVIEW development environment designed to study the real-time power quality monitoring and analysis system to achieve steady-state detecting common power quality indicators and the use of transient power quality test methods and data compression method to achieve a transient power quality real-time monitoring, the following conclusions by experimental verification:

(1) The system can achieve real-time monitoring of stationary targets, such as three-phase RMS voltage, frequency and the harmonics, etc; generalized S transform and classification rules tree 
method can achieve transient power quality disturbance signal time positioning and automatic identification .

(2) The monitoring results are accurate and error is small, stable performance, feature-rich.

(3) The system hardware design is simple, since the index in addition to the steady-state indicators, as well as transient signal to be detected, so to choose the right high-resolution data acquisition card, signal conditioning circuit design, including choice of sensors and anti-aliasing stacked low-pass filter can effectively implement the AC voltage and current signal into a voltage signal can access the data acquisition card.

(4) the system has good scalability, power quality monitoring to improve or upgrade, simply change the appropriate software modules can be, can greatly shorten the development cycle for the latter applied to substation power quality monitoring has laid a good foundation, the data provided by the monitoring system can power quality of the electricity sector governance to provide a reference.

\section{References}

[1] Wang Ling, Kang Jian, Zou Hongliang. Power System Protection and Control, Vol. 2 (2011) No 39, p.101-111

[2] Ma Jian, Sun Min, Chen Kexu. Electrical Measurement \& Instrumentation, Vol. 11 (2012) No 49, p.71-75

[3] Hang Zhou. Shaanxi Electric Power, Vol. 1 (2006) No 34, p.53-55

[4] Yang Chao. Power System Protection and Control, Vol. 5 (2012) No 40, p.21-23

[5] Zhang Gongxu, Sun Jing. Chinese CSEE, Vol. 8 (2003) No 27, p.57-60

[6] Wang Xiaohua. Chinese CSEE, Vol. 4 (2011) No 27, p. 281-289

[7] Radil T, Ramos P M, Janeiro F M, et al. Instrumentation and Measurement, IEEE Transactions on, Vol. 2 (2011) No 39, p.101-111

[8] Mishra S, Bhende C N, Panigrahi B K. IEEE Trans on Power Delivery, Vol. 11 (2012) No 49, p.71-75

[9] Feng Hao, Zhou Luowei. Power System Technology, Vol. 1 (2010) No 34, p.53-55

[10] Quan Huimin, Wang Lianhong. High Voltage Engineering, Vol. 5 (2012) No 40, p.21-23 